\title{
Congenital sick sinus syndrome caused by recessive mutations in the cardiac sodium channel gene (SCN5A)
}

\author{
D. Woodrow Benson, ${ }^{1}$ Dao W. Wang, ${ }^{2}$ Macaira Dyment, ${ }^{1}$ Timothy K. Knilans, ${ }^{1}$ \\ Frank A. Fish, ${ }^{3}$ Margaret J. Strieper, ${ }^{4}$ Thomas H. Rhodes, ${ }^{5}$ Alfred L. George, Jr. ${ }^{2,5}$ \\ ${ }^{1}$ Department of Pediatrics, Cincinnati Children's Hospital, Cincinnati, Ohio, USA \\ ${ }^{2}$ Department of Pharmacology, and \\ ${ }^{3}$ Department of Pediatrics, Vanderbilt University School of Medicine, Nashville, Tennessee, USA \\ ${ }^{4}$ Sibley Heart Center-Cardiology, Children's Healthcare of Atlanta, Atlanta, Georgia, USA \\ ${ }^{5}$ Department of Medicine, Vanderbilt University School of Medicine, Nashville, Tennessee, USA
}

\begin{abstract}
Sick sinus syndrome (SSS) describes an arrhythmia phenotype attributed to sinus node dysfunction and diagnosed by electrocardiographic demonstration of sinus bradycardia or sinus arrest. Although frequently associated with underlying heart disease and seen most often in the elderly, SSS may occur in the fetus, infant, and child without apparent cause. In this setting, SSS is presumed to be congenital. Based on prior associations with disorders of cardiac rhythm and conduction, we screened the $\alpha$ subunit of the cardiac sodium channel ( $S C N 5 A)$ as a candidate gene in ten pediatric patients from seven families who were diagnosed with congenital SSS during the first decade of life. Probands from three kindreds exhibited compound heterozygosity for six distinct $S C N 5 A$ alleles, including two mutations previously associated with dominant disorders of cardiac excitability. Biophysical characterization of the mutants using heterologously expressed recombinant human heart sodium channels demonstrate loss of function or significant impairments in channel gating (inactivation) that predict reduced myocardial excitability. Our findings reveal a molecular basis for some forms of congenital SSS and define a recessive disorder of a human heart voltage-gated sodium channel.
\end{abstract}

J. Clin. Invest. 112:1019-1028 (2003). doi:10.1172/JCI200318062.

\begin{abstract}
Introduction
Sick sinus syndrome (SSS) was described nearly 40 years ago as a complicating arrhythmia following cardioversion (1). The disorder is characterized by inappropriate sinus bradycardia, sinus arrest, or chronotropic incompetence $(2,3)$. SSS has been attributed to dysfunction of the sinoatrial (SA or sinus) node, but despite extensive efforts to define SSS in terms of abnormal automaticity, exit block, or impaired intraatrial conduction and excitability, it has remained largely an electrocardiographic diagnosis. Episodes of atrial tachycardias coexisting with sinus bradycardia (tachycardia-bradycardia syndrome) are also common in this disorder.
\end{abstract}

Received for publication February 6, 2003, and accepted in revised form July 22, 2003.

Address correspondence to: D. Woodrow Benson, Division of Cardiology, ML7042, Children's Hospital Medical Center, 3333 Burnet Avenue, Cincinnati, Ohio 45229-3039, USA Phone: (513) 636-7716; Fax: (513) 636-5958;

E-mail: woody.benson@cchmc.org.

D. Woodrow Benson and Dao W. Wang contributed equally to this work.

Conflict of interest: The authors have declared that no conflict of interest exists.

Nonstandard abbreviations used: sick sinus syndrome (SSS); human heart sodium channel (hH1); human $\beta 1$ subunit (h $\beta 1$ ); viral internal ribosome entry site (IRES); current (I); amplification-refractory mutation system analysis (ARMS); wild-type hH1 (WT-hH1); human $\beta 3$ subunit (h $\beta 3$ ); hyperpolarization-activated cyclic nucleotide-gated ( $\mathrm{HCN} 4)$.
Although primarily a disease of the elderly, SSS is an important clinical problem in pediatric patients, because treatment of associated exercise intolerance, presyncope or syncope, usually requires lifelong cardiac pacing. In children a previous history of cardiac surgery for congenital heart malformation is noted in approximately $80 \%$ of SSS cases (4-7). SSS is also seen in the fetus, infant, and child without heart disease or other contributing factors, however $(5,6,8-13)$. In this setting, a congenital origin is presumed and bradycardia progressing to atrial inexcitability or standstill has been noted, a phenomenon highly unusual in SSS associated with postoperative heart disease $(11,14)$. Familial cases consistent with autosomal dominant inheritance with reduced penetrance or recessive inheritance have been observed $(6,8,10-12,14-16)$. Familial SSS is grouped with congenital absence of sinus rhythm and familial sinus node disease under the term "nodal rhythm" in the online Mendelian Inheritance of Man database (OMIM 163800).

Based on prior associations with disorders of cardiac rhythm and conduction, we screened the $\alpha$ subunit of the cardiac sodium channel (SCN5A) as a candidate gene in ten pediatric patients from seven families who were diagnosed with congenital SSS during the first decade of life. Compound heterozygous SCN5A mutations were found in five individuals from three families, indicating that congenital SSS may, in some families, segregate as a recessive disorder of the cardiac sodium channel. 


\section{Methods}

Clinical studies

Probands with symptomatic sinus bradycardia leading to pacemaker implantation and their family members were invited to participate in the study. Informed consent was obtained from all participants in accordance with the Cincinnati Children's Hospital Medical Center Institutional Review Board. Participants were evaluated by history, review of medical records, and 12-lead electrocardiogram. Clinical studies were performed without knowledge of genotype.

\section{Molecular genetic methods}

Genomic DNA was isolated from blood of study participants, and the PCR was used to amplify the coding region and flanking intronic sequence of $S C N 5 A$, as described previously (17). Sequencing reactions were performed in the presence of fluorescence-labeled dideoxynucleotides and additional primer for exonspecific sequencing in both sense and antisense direction on isolated PCR product.

\section{Biophysical characterization of SCNSA mutants}

Mutagenesis and heterologous expression of $\mathrm{Na}$ channels. All six mutations (T220I, P1298L, G1408R, delF1617, $\mathrm{R} 1623 \mathrm{X}$, and $\mathrm{R} 1632 \mathrm{H}$ ) were engineered in a recombinant human heart sodium channel cDNA (hH1) using recombinant PCR. Final constructs were assembled in the mammalian expression plasmid pRc/CMV-hH1 and then sequenced to verify creation of the mutation and exclusion of polymerase errors.

Cells (tsA201) were transiently transfected with pRcCMV-hH1, T220I, P1298L, delF1617, or R1632H using SuperFect (QIAGEN Inc., Santa Clarita, California, USA) in combination with a plasmid (pGFP-IRES-h $\beta 1$ ) encoding both GFP and the human $\beta 1$ subunit $(\mathrm{h} \beta 1)$ under the control of a single CMV promoter with the two coding regions separated by a viral internal ribosome entry site (IRES). This approach ensures that cells exhibiting green fluorescence have been successfully transfected with the $\beta 1$-encoding plasmid. Transfections were performed with $1 \mu \mathrm{g}$ of channel-encoding plasmid DNA and $1 \mu \mathrm{g}$ of pGFP-IRES-h $\beta 1$. In some experiments, we cotransfected hH1 with pGFP-IRES$h \beta 3$ encoding the human sodium channel $\beta 3$ subunit rather than the $\beta 1$ subunit. Transiently transfected cells were transferred to a chamber (Warner Instrument Corp., Hamden, Connecticut, USA) for electrophysiological measurements 24-72 hours after transfection.

Electrophysiology. Sodium currents were recorded using the whole-cell patch-clamp technique as described previously (18). Electrode resistance ranged from 0.8 to 1.5 $\mathrm{M} \Omega$. Data acquisition was carried out using an Axopatch 200B patch-clamp amplifier and pCLAMP 8.0 software (Axon Instruments Inc., Foster City, California, USA). Currents were acquired at $20-50 \mathrm{kHz}$, filtered at $5 \mathrm{kHz}$ ( $-3 \mathrm{~dB}$, four-pole Bessel filter), and digitized using an analogue-to-digital interface (Digidata 1320A; Axon Instru- ments Inc.). Recordings from cells exhibiting peak current amplitudes less than $0.8 \mathrm{nA}$ were excluded from analysis to avoid potential endogenous channel contamination. Cells exhibiting very large whole-cell currents were also excluded if voltage control was compromised. Typically, fewer than $20 \%$ of cells were excluded from analyses. Whole-cell capacitance and access resistance were determined by integrating capacitive transients of voltage steps from $-120 \mathrm{mV}$ to $-110 \mathrm{mV}$ filtered at $10-50$ $\mathrm{kHz}$. Series resistance compensation ensured that the command potential was achieved on a microsecond time scale with voltage errors less than $3 \mathrm{mV}$. The holding potential was $-120 \mathrm{mV}$ for all experiments, except when otherwise indicated (details of each pulse protocol are given schematically in the figures and explained in Results or figure legends). The time between sequential voltage protocols was 10 seconds for all experiments. All experiments were performed at $22^{\circ} \mathrm{C}$. The bath solution contained $145 \mathrm{mM} \mathrm{NaCl}, 4 \mathrm{mM} \mathrm{KCl}, 1.8 \mathrm{mM} \mathrm{CaCl}_{2}, 1$ $\mathrm{mM} \mathrm{MgCl}_{2}, 10 \mathrm{mM}$ HEPES, and $10 \mathrm{mM}$ glucose, $\mathrm{pH} 7.35$ (adjusted with $\mathrm{NaOH}$ ). The pipette solution (intracellular solution) contained $10 \mathrm{mM} \mathrm{NaF}, 110 \mathrm{mM} \mathrm{CsF}, 20$ $\mathrm{mM}$ CsCl, 10 mM EGTA, and 10 mM HEPES, pH 7.35 (adjusted with $\mathrm{CsOH}$ ). Osmolarity was adjusted to 310 milliosmoles with sucrose for all solutions.

Data analysis. At least two independent clones were tested for each mutant, and the data were pooled. All data were analyzed using PCLAMP 8.0 (Axon Instruments Inc.), and figures were prepared using SigmaPlot 2002 (SPSS Science, Chicago, Illinois, USA). The time course of inactivation was fit with a two-exponential function: $I(t) / I_{\max }=\mathrm{A}_{1} \times \exp \left(-t / \tau_{1}\right)+\mathrm{A}_{2} \times \exp \left(-t / \tau_{2}\right)$ where values for $A$ and $\tau$ refer to amplitudes and time constants, respectively. I refers to current and $t$ refers to time. Steady-state availability was fit with the Boltzmann equation, $I / I_{\max }=\left[1+\exp \left(\left(V-V_{1 / 2}\right) / k\right)\right]-1$, to determine the membrane potential for half-maximal inactivation $\left(V_{1 / 2}\right)$ and the slope factor $k$. Recovery from inactivation was analyzed by fitting data with the twoexponential function: $I(t) / I_{\max }=\mathrm{A}_{\mathrm{f}} \times\left[1-\exp \left(-t / \tau_{\mathrm{f}}\right)\right]+\mathrm{A}_{\mathrm{s}}$ $\times\left[1-\exp \left(-t / \tau_{\mathrm{s}}\right)\right]$. Curve fitting was performed using the Marquardt-Levenberg algorithm. A coefficient of determination greater than 0.985 , standard error of each parameter less than 0.3 , and coefficients of variation less than $2 \%$ were considered evidence of the best fit. The voltage-dependence of channel activation was estimated by measuring peak sodium current during a variable test potential from a holding potential of $-120 \mathrm{mV}$. The current at each membrane potential was divided by the electrochemical driving force for sodium ions, $V_{m}-E_{\mathrm{Na}}$ ( $V_{m}$ is the test potential and $E_{\mathrm{Na}}$ represents the sodium equilibrium potential) and normalized to the maximum sodium conductance. All data were fit using a nonlinear least-squares minimization method. Results are presented as means plus or minus standard error, and the statistical comparisons were made using the unpaired Student's $t$ test. Statistical significance was assumed for $P$ values less than 0.05 . In some figures, the standard error bars are smaller than the data symbols. 


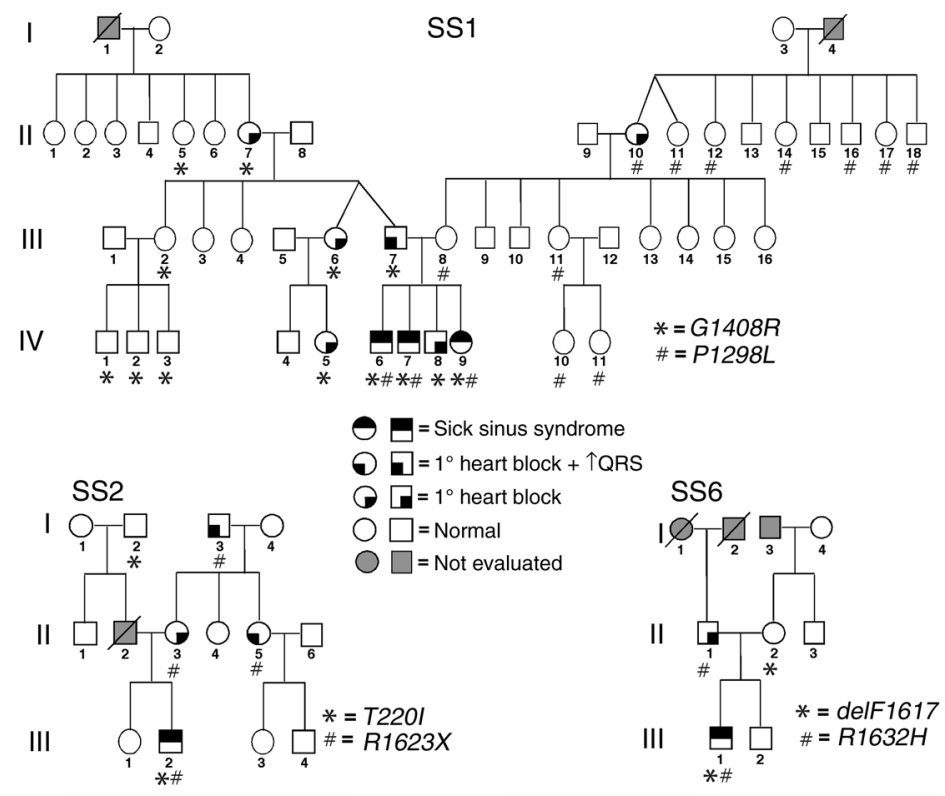

Figure 1

Congenital SSS pedigrees. Three families (SS1, SS2, SS6) with compound heterozygosity for SCN5A mutations are presented along with phenotype assignments (see symbol key) and genotypes (allele designations are indicated in the lower right of each pedigree). In the SS1 family three individuals had compound heterozygous SCN5A mutations, while in SS2 and SS6 a single individual with a compound heterozygous mutation was identified. In the three kindreds, 27 individuals were heterozygous for an SCN5A mutation, but only 10 individuals demonstrated an asymptomatic ECG phenotype of first-degree heart block (penetrance $=37 \%$ ). Slashed symbols indicate deceased individuals; Roman numerals indicate generations in the respective families.

at the time of pacemaker implant. One subject with heterotaxy syndrome died during the first month of life, but other subjects have done well for the follow-up of 2-15 years.

Compound heterozygous SCN5A mutations identified in five individuals. Based on prior associa-

\section{Results}

Clinical characteristics. Sinus bradycardia was diagnosed in ten infants and children during evaluation of abnormal heart rate; none had a history of cardiac surgery. In four cases (three families) the initial diagnosis had been made in utero (20-38 weeks gestation) during Doppler-echocardiographic evaluation of irregular fetal heart rate, and in six cases the diagnosis was made by standard electrocardiogram between the ages of two and nine years during evaluation of irregular heart rate, presyncope or syncope. A congenital heart defect, including aortic stenosis (2), pulmonary stenosis (1), or heterotaxy syndrome (1) was identified in four subjects in whom congenital SSS had been diagnosed in utero. Except for probands and their siblings, there was no family history of a cardiac rhythm disorder or other forms of cardiovascular disease in the young. Nine subjects were white and one was African-American.

All ten patients with SSS underwent pacemaker implantation. Intracardiac electrophysiology studies performed in three subjects demonstrated atrial inexcitability (no spontaneous atrial depolarization, inability to capture with pacing) and prolonged Hisventricle conduction time (60 milliseconds). Atrial inexcitability was observed in two additional patients

\section{Figure 2}

Sequencing of SCN5A mutations. (a) Automated DNA-sequencing electrophoretographs illustrating the two mutations segregating in each family (SS1, SS2, and SS6) are tiled vertically. (b) Schematic of the transmembrane topology of SCN5A illustrating the location of mutations found in congenital SSS families. Mutations are colored to indicate in which family they occur (orange circles = SS1, open circles $=$ SS2, yellow circles $=$ SS6). D1-D4 indicate homologous domains of the $\alpha$ subunit of the cardiac sodium channel. tions with disorders of cardiac rhythm and conduction, we considered $S C N 5 A$ as a candidate gene for congenital SSS. During systematic survey of all SCN5A-coding exons, compound heterozygous nucleotide changes were identified in five individuals from three kindreds (Figure 1). The age of SSS diagnosis in these five individuals was 2 years to 9 years; none had other evidence of heart disease. None of the nucleotide sequence changes were identified in over 150 chromosomes from normal, unrelated control subjects.

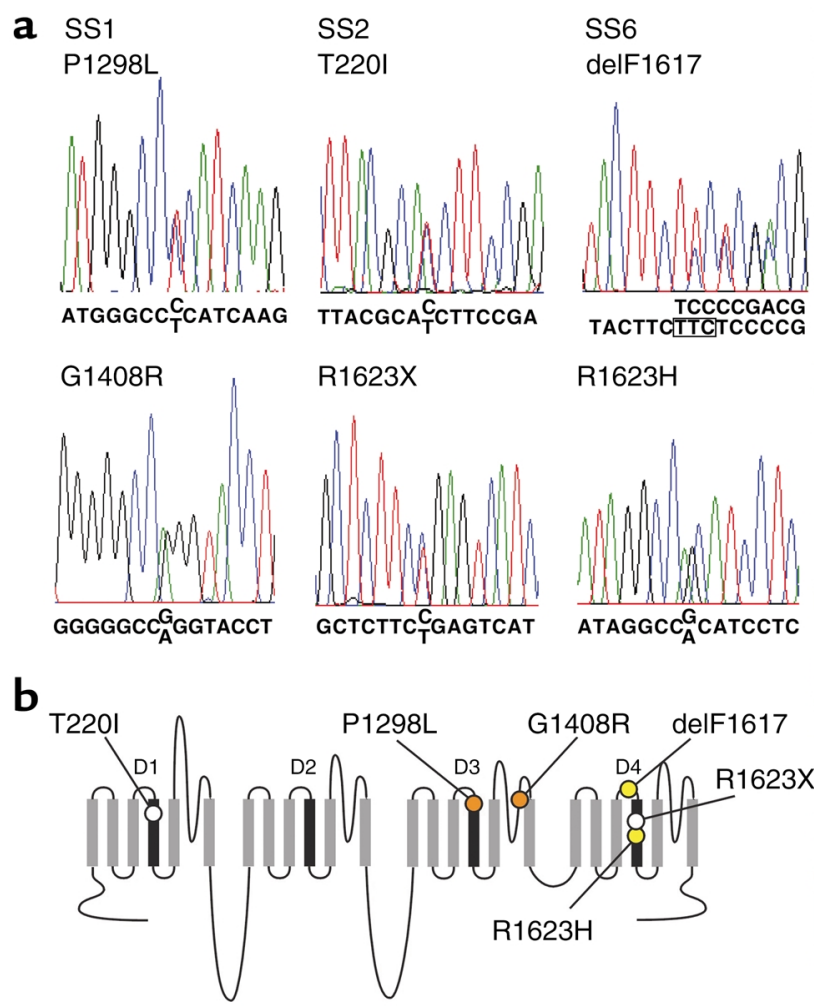


Table 1

ARMS

$\begin{array}{ll}659 \mathrm{C} \rightarrow \mathrm{T}(\mathrm{T} 220 \mathrm{I}) & \text { Primer sequence }\left(5^{\prime} \text { to } 3^{\prime}\right) \\ \text { WT F } & \text { GGACCTGGGCAATGTCTCAGCCTTACGCGC } \\ \text { Mut F } & \text { GGACCTGGGCAATGTCTCAGCCTACGCGT } \\ \text { ARMS R } & \text { TAGCCCCTGGCACCTGAGCCCTGGGAAAGG }\end{array}$

$4895 \mathrm{G} \rightarrow \mathrm{A}(\mathrm{R} 1632 \mathrm{H})$

WTF

Mut $F$

ARMS R
CCGAGTCATCCGCCTGGCCCGAATAGGCCG CCGAGTCATCCGCCTGGCCCGAATAGGCCA ACATGTTGACCACGATGAGGAAGGAGATGA
In family SS1, three individuals with congenital SSS (Figure 1, IV-6, IV-7, and IV-9) were found to have inherited two distinct missense mutations (Figure 2). The maternal allele is a previously undescribed $\mathrm{C} \rightarrow \mathrm{T}$ transition at nucleotide 3893 (nucleotide numbering based upon the open reading frame sequence of GenBank accession number M77235) and encoding leucine in place of proline 1298 (designated P1298L). A G $\rightarrow$ A transition of nucleotide 4222 was transmitted from the father and resulted in substitution of arginine for glycine at codon 1408 (G1408R). This mutation, previously designated as G1406R, was reported in a large French family segregating both Brugada syndrome and isolated cardiac conduction system disease in a domi- nant manner (19). The nucleotide changes 3893C $\rightarrow T$ and $4222 \mathrm{G} \rightarrow \mathrm{A}$ abolish a BanII site and create a BstXI restriction endonuclease site, respectively, allowing independent confirmation of P1298L and G1408R.

In family SS2 we inferred transmission to the proband (III-2) of a previously undescribed missense allele $(659 \mathrm{C} \rightarrow \mathrm{T}, \mathrm{T} 220 \mathrm{I})$ from the paternal lineage and inheritance of a novel nonsense mutation $(4867 \mathrm{C} \rightarrow \mathrm{T}$, R1623X) from his asymptomatic mother (Figure 2). Interestingly, two distinct missense mutations affecting codon 1623 (R1623Q, R1623L) have been previously associated with congenital long QT syndrome $(20,21)$. Amplification-refractory mutation system analysis (ARMS) was used to confirm the presence or absence of $659 \mathrm{C} \rightarrow \mathrm{T}$ (T220I) (Table 1) (22). The nucleotide change $4867 \mathrm{C} \rightarrow \mathrm{T}$ creates a DdeI restriction endonuclease site allowing independent confirmation of R1623X.

The proband in family SS6 (III-1) was found to have compound heterozygosity for a previously reported inframe deletion of codon 1617 (4849-4851delTTC, delF1617) inherited from the mother and a novel missense mutation $(4895 \mathrm{G} \rightarrow \mathrm{A}, \mathrm{R} 1632 \mathrm{H})$ transmitted from the father (Figure 1). Deletion of codon 1617 (del4849-4851) eliminates a single phenylalanine residue in the short extracellular loop between segments S3 and S4 in the fourth-repeat domain (D4); delF1617

\section{Figure 3}

Electrocardiographic phenotypes. (a) Lead II rhythm strip from proband (age 6 years) of family SS1 showing absent $P$ waves and prolonged QRS duration. (b and $\mathbf{c}$ ) Lead II rhythm strip and intracardiac electrophysiologic tracings from proband family SS2 (III-2) at age 9 years. No $P$ waves were evident on surface tracings, and no atrial activity was documented with intracardiac recording. Atrial pacing could not be achieved even with high output (stimuli of $7 \mathrm{~mA}, 3$ milliseconds). Both QRS duration (100 milliseconds, normal for age is less than 85 milliseconds) and His-ventricular interval ( 80 milliseconds, normal is less than 50 milliseconds) are prolonged. HBE, His bundle electrogram; $H$, potential recorded from the His bundle; RA, right atrium; RV, right ventricle. (d and $\mathbf{e}$ ) Lead II tracing from members of SS1, heterozygous for G1408R, showing (d) first-degree heart block with prolonged QRS (III-7) (e) first-degree heart block (IV-8). (f and g) In a compound heterozygote from family SS1 (IV-9), lead II tracings showing transition from first-degree heart block (f) to sinus arrest (g) at age 17 months and 25 months, respectively. (h) Lead II tracing showing first-degree heart block and prolonged QRS duration in individual II-5 family SS2, heterozygous for R1623X. A 1-second time scale is shown in c; electrocardiographic traces were obtained at standard recording conditions of $25 \mathrm{~mm} / \mathrm{s}$ and $10 \mathrm{~mm} / \mathrm{mV}$.

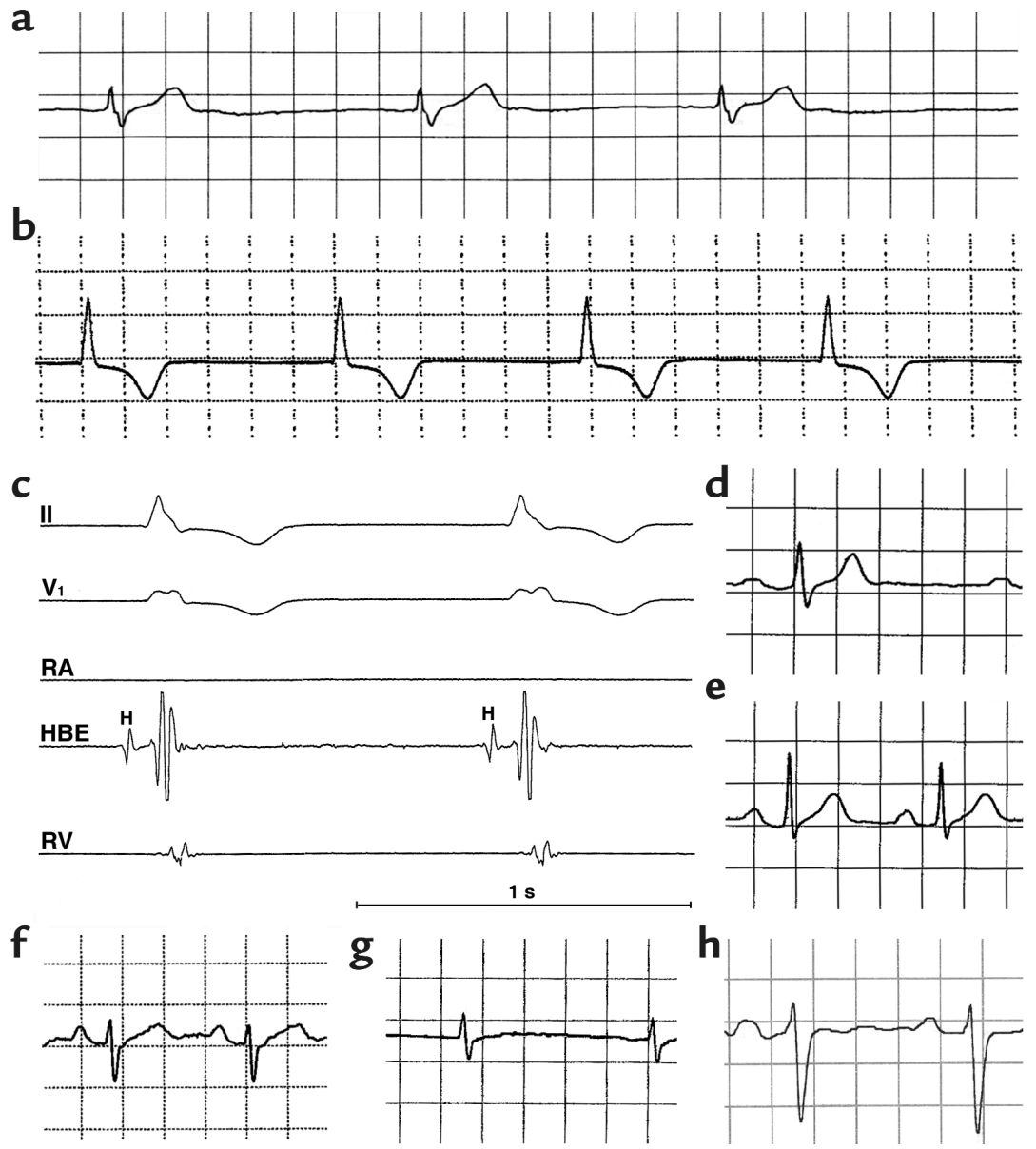


was described previously in association with congenital long QT syndrome, although no specific clinical or genetic information was provided (21). The nucleotide change $4867 \mathrm{C} \rightarrow \mathrm{T}$ abolishes a BsaXI restriction endonuclease site allowing independent confirmation of del1617F. ARMS was used to confirm the presence or absence of $4895 \mathrm{G} \rightarrow \mathrm{A}(\mathrm{R} 1632 \mathrm{H})$ (Table 1) (22).

Clinical electrophysiologic phenotype of SCN5A mutation carriers. There was no family history of cardiac arrest or sudden death in any of the three families. Among 27 heterozygotes, no individual exhibited ECG evidence of Brugada syndrome. The mean $\mathrm{QT}_{\mathrm{c}}$ in heterozygotes was $400 \pm 17$ milliseconds while that in mutation-negative family members $(n=27)$ was $423 \pm 16$ milliseconds ( $P=$ NS by Student's $t$ test).

In family SS1, electrocardiographic evaluation of bradycardia detected in the proband (IV-7) at age 6 years demonstrated absent $P$ waves, pauses up to $5 \mathrm{sec}-$ onds, and an escape rhythm at 42 beats per minute with a prolonged QRS waveform (Figure 3a). Intracardiac electrophysiologic study demonstrated a prolonged His-ventricle conduction time (72 milliseconds), absent atrial depolarizations and atrial inexcitability (not shown). Subsequent evaluation of his seven-year-old brother (IV-6) revealed similar findings. During serial observations in a younger sister (IV-9), congenital SSS was first detected at age 25 months (Figure 3, f and g). Eleven heterozygous carriers of P1298L were asymptomatic and largely without electrocardiographic abnormalities, except the maternal grandmother (II-10), who had first-degree heart block (Figure 1). Of the ten G1408R heterozygotes in family SS1 ranging in ages from 3 to 68 years, five individuals exhibited asymptomatic first-degree heart block (Figure 3, d and e), including one individual (III-7) with associated prolongation of QRS duration; the remaining five individuals had no electrocardiographic abnormalities.

During evaluation for syncope at nine years of age, the proband in family SS2 demonstrated absent $\mathrm{P}$ waves, pauses up to 15 seconds, and an escape rhythm at 50 beats per minute; intracardiac electrophysiologic study demonstrated prolongation of QRS duration and His-ventricle conduction time (68 milliseconds), absent atrial depolarizations, and atrial inexcitability (Figure 3, b and c). Following pacemaker implantation, he experienced exertional syncope. An intracardiac cardioverter defibrillator was implanted; during 10 years of treatment with $\beta$-adrenergic blockade, he has had a single discharge for an idiopathic irregular monomorphic ventricular tachycardia at $210 \mathrm{bpm}$. Three asymptomatic carriers of R1623X exhibited first-degree heart block; intraventricular conduction delay (I-3) or right bundle branch block and left anterior fascicular block (II-5, Figure $3 \mathrm{~h}$ ) was also present in one individual each. The single T220I heterozygote (I-2) in this family was clinically and electrocardiographically normal.

In family SS6, the diagnosis of congenital SSS was determined in the proband (III-1) at age 5 years. As noted in other probands, bradycardia, absent atrial depolarizations, atrial inexcitability, prolonged QRS, and prolonged His-ventricle conduction time (60 milliseconds) were observed. The single heterozygous carrier of delF1617 (II-2) had a normal electrocardiogram. The single heterozygous carrier for R1632H (II-1) demonstrated electrocardiographic features of firstdegree heart block.

Biophysical characterization of SCN5A mutants. To determine the functional consequences of the SCN5A mutations associated with congenital SSS, we engineered four missense mutations (T220I, P1298L, G1408R, $\mathrm{R} 1632 \mathrm{H})$, the in-frame deletion (delF1617), and the nonsense mutation (R1623X) in the recombinant human cardiac voltage-gated sodium channel $\alpha$ subunit (designated hH1). We then expressed each mutation heterologously in a cultured mammalian cell line (tsA201) with the sodium channel accessory h $\beta 1$ and used whole-cell patch-clamp recording to characterize resultant sodium currents. The nonsense mutation (R1623X) was suspected to encode nonfunctional channels, and mutation G1408R had previously been demonstrated to disable sodium channel function (19); our experiments confirmed these predictions (data not shown). Expression of the remaining four mutations exhibited measurable sodium channel activity in tsA201 cells and were further characterized.

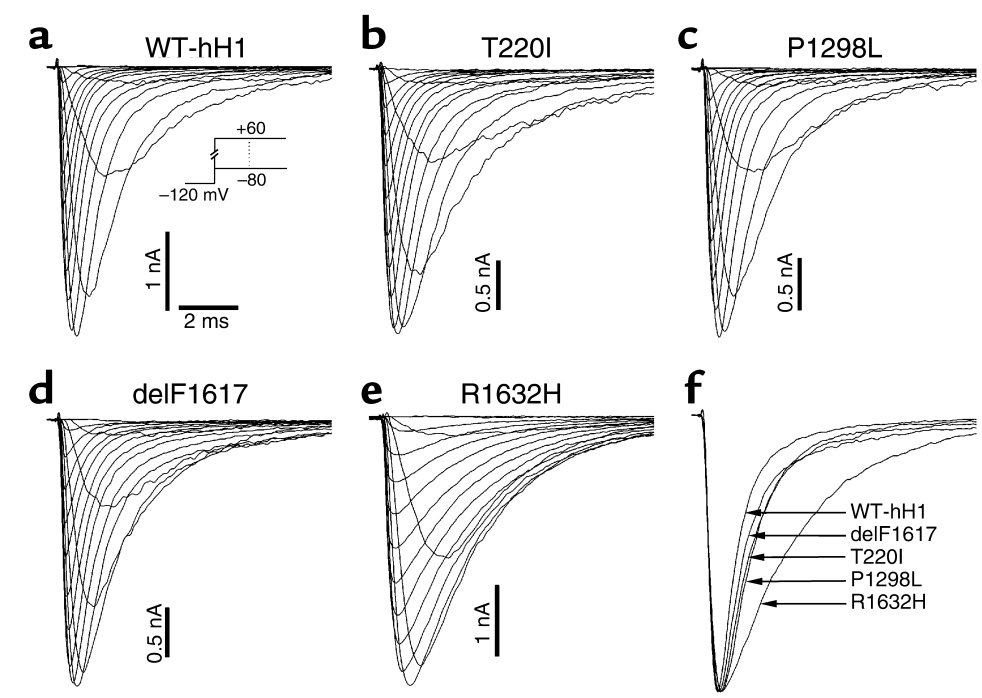

Figure 4

Whole-cell current recordings of wild-type and mutant $\mathrm{Na}$ channels. (a-e) Na channels were expressed by transient transfection in tsA201 cells in the presence of h $\beta 1$ and currents recorded at various membrane potentials from -80 to $+60 \mathrm{mV}$ in 10 $\mathrm{mV}$ increments from a holding potential of $-120 \mathrm{mV}$. (f) Normalized $\mathrm{Na}$ current at test potential of $-20 \mathrm{mV}$ for WT-hH1, T220I, P1298L, delF1617, and R1632H. 

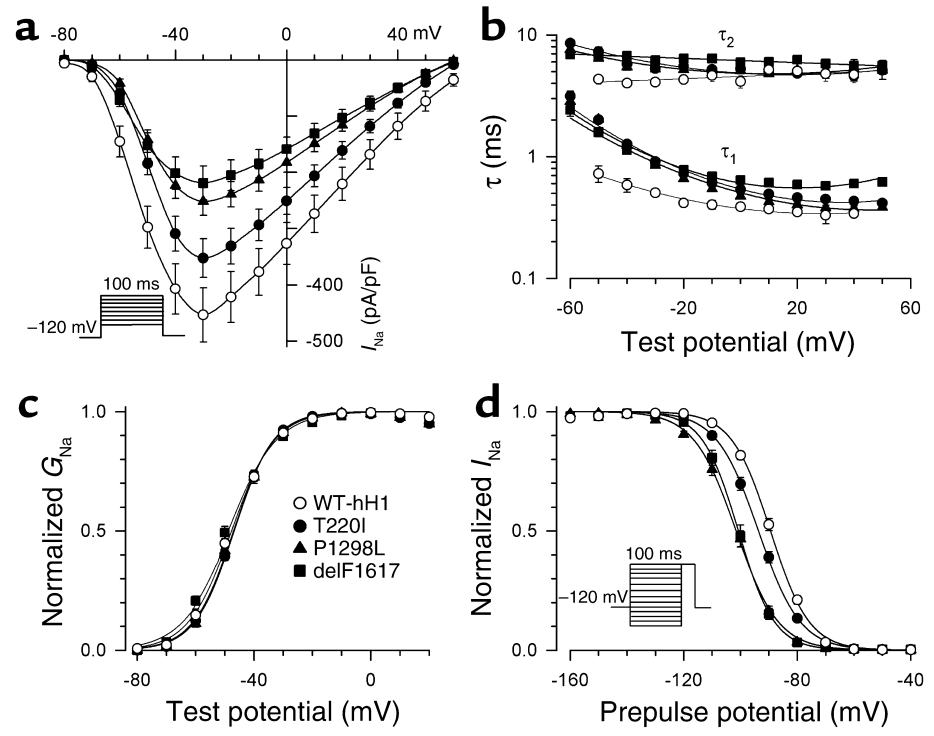

Figure 5

Impaired fast inactivation in mutant sodium channels. (a) Current-voltage relationship for WT-hH1 (open circles, $n=16$ ), T220I (filled circles, $n=25$ ), P1298L (filled triangles, $n=17$ ), and delF1617 (filled squares, $n=17$ ) sodium channels. Current (in pA) is normalized to cell capacitance (in picofarads, $\mathrm{pF}$ ) to give a measure of sodium current density. Current density is significantly lower for T220I, P1298L, and delF1617 at test potentials between $-60 \mathrm{mV}$ and $+60 \mathrm{mV}$ $(P<0.05)$. (b) Voltage dependence of fast inactivation time constants $\left(\tau_{1}\right.$ and $\tau_{2}$ ) for WT-hH1 (open circles, $n=16$ ), T220I (filled circles, $n=25$ ), P1298L (filled triangles, $n=17$ ), and delF1617 (filled squares, $n=17$ ). Lower and upper bundles of symbols indicate $\tau_{1}$ and $\tau_{2}$ values, respectively. Differences between WT$\mathrm{hH} 1$ and mutant channels were significant for $\tau_{1}$ (T220I and P1298L; $P<0.05$ at tested voltage between -50 to $-10 \mathrm{mV}$; delF1617, $P<0.05$ at all tested voltages). In some cases, error bars are smaller than the data symbol. (c) Voltage dependence of activation for WT-hH1 (open circles), T220I (filled circles), P1298L (filled triangles), and delF1617 (filled squares). Curves were fit with a Boltzmann distribution, and values determined for the voltage midpoint $\left(V_{1 / 2}\right)$ and slope factor $(k)$ are shown in Table 2. G, conductance. (d) Sodium channel availability for WT-hH1 $(n=19)$, T220I $(n=30), \mathrm{P} 1298 \mathrm{~L}(n=32)$, and delF1617 $(n=12)$ recorded using the pulse protocol shown in the inset and fit with Boltzmann distributions (solid lines). The half-maximal voltage for $\mathrm{Na}$ channel inactivation $\left(V_{1 / 2}\right)$ and slope factor are listed in Table 2.

Figure 4 illustrates representative whole-cell current tracings from cells expressing either wild-type hH1 (WT-hH1), T220I, P1298L, delF1617, or R1632H. All recombinant alleles exhibited rapid activation and inactivation in response to a series of depolarizing test potentials typical of voltage-gated sodium channels. The mutants differed from WT-hH1, however, in the time course of inactivation. Three of the mutations (T220I, P1298L, delF1617) shared a similar mild degree of inactivation delay while $\mathrm{R} 1632 \mathrm{H}$ exhibited a more severe impairment (Figure 4f). Given the similarity of the biophysical disturbance exhibited by T220I, P1298L, and delF1617, we present the functional characterization of these mutations separately from that of $\mathrm{R} 1632 \mathrm{H}$.

In Figure 5, additional studies demonstrated a constellation of biophysical defects shared by T220I, P1298L, and delF1617, including reduced peak sodium current density (Figure $5 a)$, impaired fast inactivation (Figure 5b), normal voltage dependence of activation (Figure 5c), and hyperpolarizing shifts in the voltage dependence of steady-state channel availability (Figure 5d). The delayed inactivation exhibited by these mutations is expected to produce enhanced sodium current over time and could be considered a gain-of-function defect. Decreased peak sodium current density and the hyperpolarized shift in channel availability, however, will reduce sodium channel activity for the mutants. In addition to these findings, T220I, P1298L, and delF1617 substantially impair the rate at which channels recover from inactivation (Figure 6). This phenomenon will likely reduce channel function by lengthening the refractory period following channel activation. Overall, the combination of biophysical characteristics we observed for T220I, P1298L, and delF1617 would indicate a net loss of sodium channel function.

For R1632H, we observed profound abnormalities in channel function that shared similarities with the other mutants, except that $\mathrm{R} 1632 \mathrm{H}$ channels did not exhibit reduced sodium current densities (Figure 7a). R1632H channels have a severe impairment in fast inactivation as illustrated by the voltage dependence of the inactivation time constants in Figure $7 \mathrm{~b}$. Steady-state channel availability exhibited a hyperpolarized voltage dependence that required a more negative holding

Table 2

Biophysical parameters for activation and inactivation in the presence of $h \beta 1$

\begin{tabular}{|c|c|c|c|c|c|c|c|c|}
\hline & \multicolumn{3}{|c|}{$\begin{array}{l}\text { Voltage dependence } \\
\text { of activation }\end{array}$} & \multicolumn{3}{|c|}{ Steady-state availability } & \multicolumn{2}{|c|}{ Recovery from inactivation } \\
\hline & $V_{1 / 2}(\mathrm{mV})$ & $k(\mathrm{mV})$ & $n$ & $V_{1 / 2}(\mathrm{mV})$ & $k(\mathrm{mV})$ & $n$ & $\tau_{1}(\mathrm{~ms})$ (amplitude, $\left.\%\right)$ & $\tau_{2}(\mathrm{~ms})$ (amplitude, $\left.\%\right)$ \\
\hline WT-hH1 & $-47.7 \pm 1.1$ & $7.0 \pm 0.2$ & 16 & $-89.4 \pm 0.7$ & $-6.5 \pm 0.1$ & 19 & $4.3 \pm 0.3(96.8 \pm 1.5)$ & $58.3 \pm 6.8(3.2 \pm 1.5)$ \\
\hline T220I & $-46.8 \pm 0.6$ & $6.7 \pm 0.2$ & 25 & $-93.8 \pm 0.9^{\mathrm{A}}$ & $-6.9 \pm 0.1$ & 30 & $10.8 \pm 0.8^{B}(96.9 \pm 0.7)$ & $191.0 \pm 26.9^{\mathrm{B}}(3.1 \pm 0.7)$ \\
\hline P1298L & $-46.5 \pm 0.9$ & $6.8 \pm 0.2$ & 17 & $-101.7 \pm 1.0^{B}$ & $-6.7 \pm 0.2$ & 32 & $12.5 \pm 1.3^{\mathrm{B}}(95.8 \pm 0.7)$ & $279.2 \pm 47.7^{\mathrm{B}}(4.2 \pm 0.7)$ \\
\hline delF1617 & $-48.8 \pm 1.0$ & $8.0 \pm 0.2$ & 17 & $-100.8 \pm 1.2^{\mathrm{B}}$ & $-6.0 \pm 0.2$ & 12 & $9.3 \pm 1.1^{\mathrm{B}}(94.7 \pm 1.4)$ & $160.0 \pm 36.0^{\mathrm{B}}(5.3 \pm 1.4)$ \\
\hline $\mathrm{R} 1632 \mathrm{H}$ & $-46.4 \pm 0.7$ & $6.4 \pm 0.2$ & 25 & $-104.0 \pm 2.0^{\mathrm{B}}$ & $-7.0 \pm 0.2$ & 15 & $135.7 \pm 13.2^{\mathrm{B}}(34.1 \pm 1.6)^{\mathrm{B}}$ & $611.1 \pm 42.9^{B}(65.9 \pm 1.6)^{B}$ \\
\hline
\end{tabular}

${ }^{A} P<0.001 ;{ }^{B} P<0.0001$. 


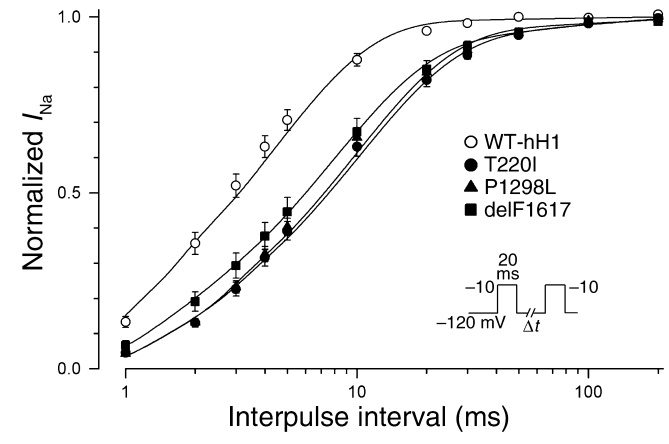

Figure 6

SCN5A mutants exhibit impaired recovery from inactivation. The time course of recovery from inactivation was elicited using the twopulse protocol shown in the inset. Time constants and fractional amplitudes are listed in Table 2.

potential $(-140 \mathrm{mV}$ versus $-120 \mathrm{mV}$ used in characterizing the other mutants) to achieve steady-state inactivation (Figure 7c). Furthermore, R1632H channels never activated when the holding potential was $-90 \mathrm{mV}$. As observed with the other three alleles, the voltage dependence of activation was not different from WThH1 (Figure 7c). Recovery from inactivation, however, was profoundly impaired in R1632H channels (Figure 7d) with complete restoration of channel availability achieved only after 4,000 milliseconds. This dramatic defect predicts that mutant channels will have insufficient time to recover from inactivation during a normal diastolic interval. Consistent with this notion, we observed rapid attenuation of R1632H channel availability during repetitive depolarizations (stepping to $-10 \mathrm{mV}$ from $-120 \mathrm{mV}$ for 500 milliseconds at 1 $\mathrm{Hz}$ ) designed to simulate a normal heart rate (data not shown). Under the same conditions, there was no loss of availability for WT channels. Overall, the pattern of biophysical defects observed for $\mathrm{R} 1632 \mathrm{H}$ is best characterized as a mixture of gain (delayed inactivation) and loss (hyperpolarized voltage-dependence of steady-state channel availability and delayed recovery) of function with a predominance of functional loss.

In light of the observation that myocardial conduction appears affected to a greater degree in the atria, we considered the possibility that an accessory protein expressed only in the ventricle normalizes function of the mutant sodium channels. Evidence exists that the sodium channel $\beta 3$ subunit is restricted to the ventricle (23), and therefore we tested whether coexpression of a human $\beta 3$ subunit $(\mathrm{h} \beta 3)$ alters functional properties of the SCN5A

\section{Figure 7}

mutants. With few exceptions, the biophysical properties of WT-hH1 and the four mutants coexpressed with $h \beta 3$ were highly similar to the condition of $h \beta 1$ coexpression (Table 3 ). Coexpression of $h \beta 3$, however, does shift steady-state availability for WT-hH1 to more depolarizing potentials that predict enhanced channel activity in the ventricles, an effect partially offset by the significant depolarizing shift in the voltage-dependence of activation. Coexpression of $h \beta 3$ did not exhibit a similar effect on any of the mutant sodium channels, however. By contrast, $\mathrm{h} \beta 3$ shifts steady-state availability of T220I to more negative potentials. Recovery from inactivation of WT-hH1, T220I, and P1298L in the presence of $h \beta 3$ exhibited a monoexponential time course rather than the double-exponential recovery kinetics observed with $h \beta 1$. We did observe a significant difference in the $\tau_{2}$ recovery time constant for delF1617 in the presence of $h \beta 3$, but this represents only a minor population of channels (6.6\%; Table 3$)$. The overall time course of recovery from inactivation was not greatly different for any mutant in the presence of $h \beta 3$ compared with $h \beta 1$. Based upon these observations, we cannot attribute the apparent milder ventricular phenotype to the $\beta 3$ subunit.
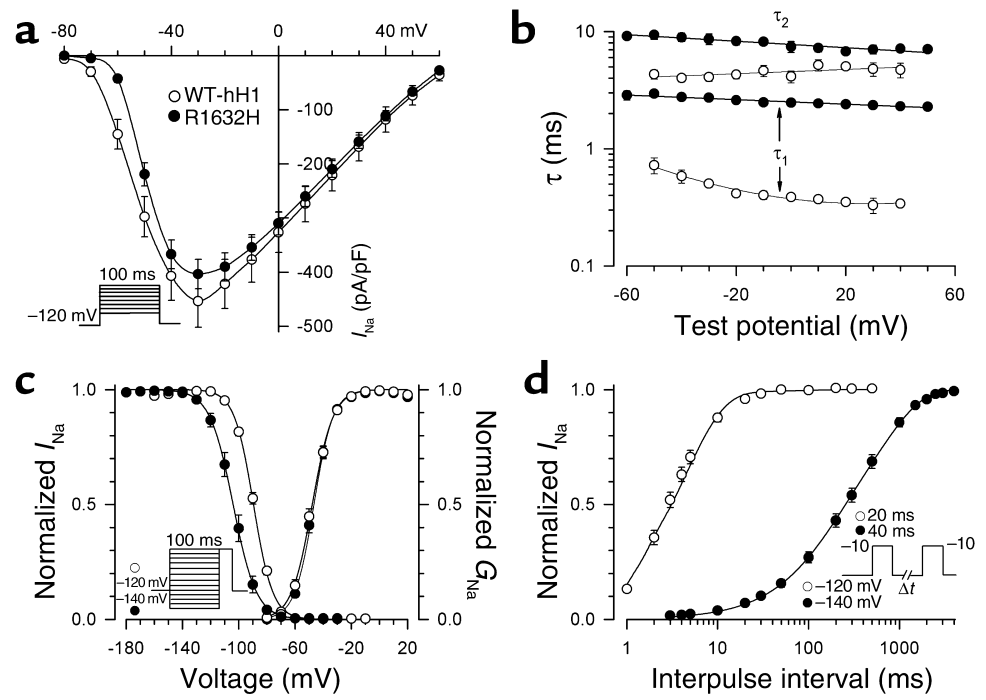

Biophysical properties of $\mathrm{R} 1632 \mathrm{H}$. (a) Comparison of current-voltage relationship for WT-hH1 (open circles) and R1632H (filled circles, $n=25$ ). Current is normalized to cell capacitance to give a measure of sodium current density. There is no difference in current density between WT-hH1 and $\mathrm{R} 1632 \mathrm{H}$ at all tested voltages. (b) Voltage dependence of fast inactivation time constants for WT-hH1 (open circles) and $\mathrm{R} 1632 \mathrm{H}$ (filled circles, $n=25$ ). Differences between WT-hH1 and mutant channel were significant for $\tau_{1}(P<0.0001)$ and $\tau_{2}(P<0.05)$ at voltages between -60 to +50 $\mathrm{mV}$. (c) Voltage dependence of sodium channel availability and activation (symbol definitions are shown as an inset, and their shading patterns are explained in the $y$-axis labels). Voltage dependence of sodium channel availability (steady-state inactivation) was obtained using a two-pulse protocol as illustrated by the inset. The membrane potentials for half-maximal inactivation and slope factors are provided in Table 2. The activation curve was constructed as described in the legend of Figure 5, and parameters are given in Table 2. (d) Time course of recovery from inactivation at $-120 \mathrm{mV}(-140 \mathrm{mV}$ for $\mathrm{R} 1632 \mathrm{H})$. The time constants and fractional amplitudes (given in parentheses) are provided in Table 2. 
Biophysical parameters for activation and inactivation in the presence of $\mathrm{h} \beta 3$

\begin{tabular}{|c|c|c|c|c|c|c|c|c|c|}
\hline & \multicolumn{3}{|c|}{ Voltage dependence of activation } & \multicolumn{3}{|c|}{ Steady-state availability } & \multicolumn{3}{|c|}{ Recovery from inactivation } \\
\hline & $V_{1 / 2}(\mathrm{mV})$ & $k(\mathrm{mV})$ & $n$ & $V_{1 / 2}(\mathrm{mV})$ & $k(\mathrm{mV})$ & $n$ & $\begin{array}{c}\tau_{1}(\mathrm{~ms}) \\
\text { (amplitude, \%) }\end{array}$ & $\begin{array}{c}\tau_{2}(\mathrm{~ms}) \\
\text { (amplitude, \%) }\end{array}$ & $n$ \\
\hline WT-hH1 & $-39.3 \pm 0.8^{B}$ & $6.3 \pm 0.4$ & 9 & $-82.3 \pm 0.6^{\mathrm{B}}$ & $-6.4 \pm 0.1$ & 10 & $5.1 \pm 0.3$ & NA & 10 \\
\hline T220I & $-45.9 \pm 1.4$ & $8.2 \pm 0.4^{B}$ & 12 & $-97.6 \pm 1.4^{\mathrm{A}}$ & $-7.1 \pm 0.2$ & 12 & $16.4 \pm 1.7$ & NA & 11 \\
\hline P1298L & $-46.5 \pm 1.5$ & $7.1 \pm 0.6$ & 13 & $-100.4 \pm 1.0$ & $-6.0 \pm 0.1^{\mathrm{A}}$ & 13 & $10.5 \pm 0.8$ & NA & 13 \\
\hline delF1617 & $-50.4 \pm 2.7$ & $8.3 \pm 0.7$ & 10 & $-104.3 \pm 1.5$ & $-5.9 \pm 0.2$ & 14 & $\begin{array}{c}11.3 \pm 1.3 \\
93.4 \% \pm 1.6 \%\end{array}$ & $\begin{array}{l}70.7 \pm 10.1^{\mathrm{A}} \\
6.6 \% \pm 1.6 \%\end{array}$ & 14 \\
\hline $\mathrm{R} 1632 \mathrm{H}$ & $-44.5 \pm 1.5$ & $7.4 \pm 0.3^{\mathrm{A}}$ & 10 & $-99.1 \pm 1.5$ & $-6.1 \pm 0.1^{B}$ & 11 & $\begin{aligned} 199.1 & \pm 38 \\
41.1 \% & \pm 4.6 \%\end{aligned}$ & $\begin{array}{l}489.6 \pm 58.0 \\
58.9 \% \pm 4.6 \%\end{array}$ & 11 \\
\hline
\end{tabular}

${ }^{A} P<0.05 ;{ }^{B} P<0.001$ compared with coexpression with $\mathrm{h} \beta 1$ (Table 2).

\section{Discussion}

Voltage-gated sodium channels are responsible for the generation and propagation of action potentials in most excitable tissues (24). In the heart, sodium channels are essential for the orderly progression of action potentials from the sinoatrial node, through atrial myocardium, across the atrioventricular node, along the specialized conduction system of the ventricles (including the Purkinje fibers), and ultimately throughout ventricular myocardium to enable rhythmic pumping of blood throughout the circulation (25). Intrinsic disturbances in the electrical excitability of cardiac tissues can elicit life-threatening disorders of heart rhythm (arrhythmias) (26).

Inherited arrhythmias associated with mutations in the cardiac sodium channel $\alpha$ subunit gene include three distinct "channelopathies" (27). Congenital long QT syndrome (28), idiopathic ventricular fibrillation (including Brugada syndrome as well as sudden unexplained death syndrome) $(29,30)$, and progressive cardiac conduction system disease $(18,31,32)$ are all dominant disorders of $S C N 5 \mathrm{~A}$. The clinical heterogeneity associated with $S C N 5 A$ mutations is partly explained by corresponding differences in the degree and characteristics of sodium channel dysfunction. In congenital long QT syndrome (LQT3), biophysical defects in the SCN5A protein lead to a dominant gain-of-function phenotype at the molecular level (33). By contrast, Brugada syndrome and conduction system disease results from predominantly loss-of-function defects in this sodium channel (18, 32, 34-36). Haploinsufficiency may explain the phenotype associated with the latter disorders in light of the recent demonstration of arrhythmias and conduction block in mice heterozygous for a $\operatorname{Scn} 5$ a null allele, whereas complete absence of $S c n 5 a$ in mice results in embryonic lethality (37).

Here we demonstrate that SCN5A mutations explain a recessive disorder of cardiac conduction characterized by bradycardia progressing to atrial inexcitability during the first decade of life. Affected individuals also manifest abnormal ventricular depolarization (prolonged QRS) and delayed His-ventricle conduction. The cardiac sodium channel $\alpha$ subunit was considered a candidate gene for this condition because of the frequent occurrence of bradycardia in carriers of dominant $L Q T 3$ mutations and the occurrence of SCN5A-associated conduction system disease. In three families transmission of the phenotype was autosomal recessive with complete penetrance. Mutation carriers were asymptomatic, but some exhibited subclinical evidence of a latent cardiac conduction system disease (i.e., first degree atrioventricular block). Evidence for subclinical organ dysfunction is not uncommon among recessively inherited conditions, including some channelopathies. For example, in the skeletal muscle disorder recessive generalized myotonia, latent myotonia may be evident on electromyography in carriers of recessive chloride channel mutations (38). Interestingly, two mutations segregating as recessive alleles in our families have been identified previously in association with dominant syndromes (delF1617 in LQTS; G1408R in Brugada syndrome with conduction system disease) $(19,21)$. The other four alleles were not associated previously with dominant inherited arrhythmia syndromes and may represent exclusively recessive $S C N 5 A$ mutations.

Two of the six mutations associated with congenital SSS have been demonstrated to produce nonfunctional sodium channels (G1408R, R1623X). The remaining alleles were functional when expressed heterologously in cultured mammalian cells and exhibited a similar pattern of biophysical abnormalities characterized by impaired inactivation and slowed recovery from inactivation. Three mutations exhibited mild to moderate dysfunction (T220I, P1298L, delF1617), while the R1632H allele was more severely impaired. The latter allele exhibits a profound defect in recovery from inactivation, suggesting that sodium channels with this mutation will have severe activity-dependent loss of availability. Interestingly, the three compound genotypes associated with congenital SSS observed in these families are paired to include one nonfunctional or severely impaired allele in combination with a mutation having a milder biophysical phenotype (Figure 2). This observation predicts that affected individuals have residual, albeit dysfunctional, myocardial sodium channel activity.

We believe that congenital SSS is the first recognized recessive disorder of the cardiac sodium channel. 
Lupoglazoff et al., however, described a child homozygous for a missense SCN5A allele (V1777M) who exhibited LQTS with rate-dependent atrioventricular conduction block (39). The child's parents and one heterozygous sibling had borderline QT interval prolongation but were asymptomatic. Biophysical studies of the $\mathrm{V} 1777 \mathrm{M}$ variant demonstrated noninactivating sodium current resembling the defect observed with most LQTS-associated SCN5A mutations. These data are most consistent with recessive inheritance of an otherwise dominant mutation exhibiting reduced penetrance in the heterozygous parents and sibling.

The current study and two recent reports clearly establish the genetic heterogeneity associated with familial sinus bradycardia syndromes. For example, atrial bradycardia, atrial standstill, prolonged His-ventricle conduction time, and age of onset in the third or fourth decade in a small Dutch kindred was attributed to the coinheritance of a heterozygous SCN5A mutation (D1275N) and a specific haplotype in the connexin-40 promoter (40). Failure of the SCN5A allele alone to confer a clinical phenotype supported an argument favoring digenic inheritance. The biophysical properties of D1275N, however, were distinct from that observed in the four functional alleles we studied. These mutants exhibited small differences in the voltage-dependence of activation observed in the absence of the $\beta 1$ accessory subunit and acceleration of recovery from inactivation. Schulze-Bahr et al. reported a mutation in a component of $\mathrm{I}_{f}$ current (hyperpolarization-activated cyclic nucleotide gated, or HCN4) in a sporadic case of pacemaker dysfunction characterized by bradyarrhythmia, chronotropic incompetence, and intermittent atrial fibrillation with age of onset in the fifth decade (41). Biophysical studies suggested a dominant negative effect of the HCN4 mutant on wild-type channels; If like currents with altered biophysical properties were identified. Taken together, these findings suggest that distinct genetic and molecular mechanisms exist to explain sinus bradycardia.

We can speculate on the pathogenesis of congenital SSS associated with recessive SCN5A mutations. Primary dysfunction of the sinoatrial node seems unlikely because action potentials in this tissue are largely calcium channel dependent or are associated with tetrodotoxin-sensitive (TTX-sensitive) sodium current in contrast to the TTX-resistant current arising from the sodium channel encoded by SCN5A (42). Sinus node dysfunction caused by failure of impulses to conduct into adjacent atrial myocardium (exit block) has been suggested as a cause of acquired SSS $(43,44)$ and is a plausible mechanism to explain this $S C N 5 A$-linked disorder. The clinical progression from bradycardia to atrial inexcitability further suggests that factors associated with postnatal development may contribute to the emergence of the complete phenotype. Although atrial action potentials progressively disappear in this disorder and intraventricular conduction is abnormal, a ventricular escape rhythm was evident in affected members of these families. This implies that action potential generation and/or propagation is more severely impaired in the atria than ventricles. It is conceivable that intrinsic differences between atrial and ventricular myocardium, such as liminal length for action potential propagation or sodium current densities, may underlie the more severe atrial phenotype. We can also speculate that the generation of ventricular action potentials in this disorder might depend upon sodium channel isoforms other than SCN5A expressed in ventricular tissue $(45,46)$. Alternatively, ventriclespecific metabolic or signal transduction events and alternative processing of SCN5A mRNA or accessory proteins other than $\beta 1$ and $\beta 3$ subunits may enable partial rescue of mutant $S C N 5 A$ phenotypes in the ventricle but not the atrial myocardium.

\section{Acknowledgments}

The authors are grateful to the families who participated in this study. The studies were facilitated by the assistance of Linda Boehm, Burt I. Bromberg, Kerry Howell, Connie Jones, James Joyce, and Peter Karpawich in collecting patient material. We also thank the Vanderbilt DNA Sequencing Facility and the Cincinnati Children's Hospital Medical Center Genomics Core Facility. This work was supported by the NIH (grant HD-39946 to D.W. Benson, grant NS-32387 to A.L. George, Jr.) and the American Heart Association Southeast Affiliate (grant AHA-0160241B to D.W. Wang). We thank Dan Roden, John D. Kugler, and Frank Zimmerman for critical reading of the manuscript.

1. Lown, B. 1967. Electrical reversion of cardiac arrhythmias. Br. Heart J. 29:469-489.

2. Fish, F.A., and Benson, D.W. 2001. Disorders of cardiac rhythm and conduction. In Moss and Adam's heart disease in infants, children and adolescents. H.D. Allen, E.B. Clark, H.P. Gutgesell, and D.J. Driscoll, editors. Lippincott, Williams \& Wilkins. Philadelphia, Pennsylvania, USA. 482-533.

3. Martin, A.B., and Kugler, J.D. 1999. Sinus node dysfunction. In Clinical pediatric arrbythmias: electrophysiology and pacing. P.C. Gillette and A.J. Garson, editors. W.B. Saunders. Philadelphia, Pennsylvania, USA. 51-62.

4. Yabek, S.M., Swensson, R.E., and Jarmakani, J.M. 1977. Electrocardiographic recognition of sinus node dysfunction in children and young adults. Circulation. 56:235-239.

5. Yabek, S.M., and Jarmakani, J.M. 1978. Sinus node dysfunction in children, adolescents, and young adults. Pediatrics. 61:593-598.

6. Radford, D.J., and Izukawa, T. 1975. Sick sinus syndrome. Symptomatic cases in children. Arch. Dis. Child. 50:879-885.

7. Kugler, J.D., Gillette, P.C., Mullins, C.E., and McNamara, D.G. 1979 Sinoatrial conduction in children: an index of sinoatrial node function. Circulation. 59:1266-1276.

8. Ector, H., and Van der Hauwaert, L.G. 1980. Sick sinus syndrome in childhood. Br. Heart J. 44:684-691.

9. Beder, S.D., Gillette, P.C., Garson, A., Jr., Porter, C.B., and McNamara, D.G. 1983. Symptomatic sick sinus syndrome in children and adolescents as the only manifestation of cardiac abnormality or associated with unoperated congenital heart disease. Am. J. Cardiol. 51:1133-1136.

10. Guillerm, F., et al. 1989. Sick sinus syndrome in children with a "healthy heart". Apropos of 2 cases with direct endocavitory tracing of the sinoatrial block [in French.]. Ann. Cardiol. Angeiol. (Paris). 38:143-146.

11. Nordenberg, A., Varghese, P.J., and Nugent, E.W. 1976. Spectrum of sinus node dysfunction in two siblings. Am. Heart J. 91:507-512.

12. Scott, O., Macartney, F.J., and Deverall, P.B. 1976. Sick sinus syndrome in children. Arch. Dis. Child. 51:100-105.

13. Yabek, S.M., Dillon, T., Berman, W., Jr., and Niland, C.J. 1982. Symptomatic sinus node dysfunction in children without structural heart disease. Pediatrics. 69:590-593.

14. Ward, D.E., Ho, S.Y., and Shinebourne, E.A. 1984. Familial atrial standstill and inexcitability in childhood. Am. J. Cardiol. 53:965-967. 
15. Spellberg, R.D. 1971. Familial sinus node disease. Chest. 60:246-251.

16. Bharati, S., Surawicz, B., Vidaillet, H.J., Jr., and Lev, M. 1992. Familial congenital sinus rhythm anomalies: clinical and pathological correlations. Pacing Clin. Electrophysiol. 15:1720-1729.

17. Wang, Q., Li, Z., Shen, J., and Keating, M.T. 1996. Genomic organization of the human SCN5A gene encoding the cardiac sodium channel. Genomics. 34:9-16.

18. Wang, D.W., Viswanathan, P.C., Balser, J.R., George, A.L., Jr., and Benson, D.W. 2002. Clinical, genetic, and biophysical characterization of SCN5A mutations associated with atrioventricular conduction block. Circulation. 105:341-346.

19. Kyndt, F., et al. 2001. Novel SCN5A mutation leading either to isolated cardiac conduction defect or Brugada syndrome in a large French family. Circulation. 104:3081-3086.

20. Makita, N., et al. 1998. A de novo missense mutation of human cardiac $\mathrm{Na}+$ channel exhibiting novel molecular mechanisms of long QT syndrome. FEBS Lett. 423:5-9.

21. Splawski, I., et al. 2000. Spectrum of mutations in long-QT syndrome genes. KVLQT1, HERG, SCN5A, KCNE1, and KCNE2. Circulation. 102:1178-1185.

22. Little, S. 1995. Current protocols in human genetics. John Wiley and Sons. New York, New York, USA. 9.8.1-9.8.6.

23. Fahmi, A.I., et al. 2001. The sodium channel beta-subunit SCN3b modulates the kinetics of SCN5a and is expressed heterogeneously in sheep heart. J. Physiol. 537:693-700.

24. Catterall, W.A. 2000. From ionic currents to molecular mechanisms: the structure and function of voltage-gated sodium channels. Neuron. 26:13-25.

25. Balser, J.R. 1999. Structure and function of the cardiac sodium channels. Cardiovasc. Res. 42:327-338.

26. Roberts, R., and Brugada, R. 2003. Genetics and arrhythmias. Annu. Rev Med. 54:257-267.

27. Marban, E. 2002. Cardiac channelopathies. Nature. 415:213-218.

28. Wang, Q., et al. 1995. Cardiac sodium channel mutations in patients with long QT syndrome, an inherited cardiac arrhythmia. Hum. Mol. Genet. 4:1603-1607.

29. Wang, Q., et al. 1995. SCN5A mutations associated with an inherited cardiac arrhythmia, long QT syndrome. Cell. 80:805-811.

30. Vatta, M., et al. 2002. Genetic and biophysical basis of sudden unexplained nocturnal death syndrome (SUNDS), a disease allelic to Brugada syndrome. Hum. Mol. Genet. 11:337-345.

31. Schott, J.J., et al. 1999. Cardiac conduction defects associate with mutations in SCN5A. Nat. Genet. 23:20-21.
32. Tan, H.L., et al. 2001. A sodium-channel mutation causes isolated cardiac conduction disease. Nature. 409:1043-1047.

33. Bennett, P.B., Yazawa, K., Makita, N., and George, A.L., Jr. 1995. Molecular mechanism for an inherited cardiac arrhythmia. Nature. 376:683-685.

34. Dumaine, R., et al. 1999. Ionic mechanisms responsible for the electrocardiographic phenotype of the Brugada syndrome are temperature dependent. Circ. Res. 85:803-809.

35. Deschenes, I., et al. 2000. Electrophysiological characterization of SCN5A mutations causing long QT (E1784K) and Brugada (R1512W and R1432G) syndromes. Cardiovasc. Res. 46:55-65.

36. Wang, D.W., Makita, N., Kitabatake, A., Balser, J.R., and George, A.L., Jr. 2000. Enhanced $\mathrm{Na}(+)$ channel intermediate inactivation in Brugada syndrome. Circ. Res. 87:E37-E43.

37. Papadatos, G.A., et al. 2002. Slowed conduction and ventricular tachycardia after targeted disruption of the cardiac sodium channel gene Scn5a. Proc. Natl. Acad. Sci. U. S. A. 99:6210-6215.

38. Becker, P.E. 1979. Heterozygote manifestation in recessive generalized myotonia. Hum. Genet. 46:325-329.

39. Lupoglazoff, J.M., et al. 2001. Homozygous SCN5A mutation in long-QT syndrome with functional two-to-one atrioventricular block. Circ. Res. 89:E16-E21.

40. Groenewegen, W.A., et al. 2003. A cardiac sodium channel mutation cosegregates with a rare connexin 40 genotype in familial atrial standstill. Circ. Res. 92:14-22.

41. Schulze-Bahr, E., et al. 2003. Pacemaker channel dysfunction in a patient with sinus node disease. J. Clin. Invest. 111:1537-1545. doi:10.1172/ JCI200316387.

42. Gellens, M.E., et al. 1992. Primary structure and functional expression of the human cardiac tetrodotoxin-insensitive voltage-dependent sodium channel. Proc. Natl. Acad. Sci. U. S. A. 89:554-558.

43. Asseman, P., et al. 1983. Persistent sinus nodal electrograms during abnormally prolonged postpacing atrial pauses in sick sinus syndrome in humans: sinoatrial block vs. overdrive suppression. Circulation. 68:33-41.

44. Asseman, P., et al. 1991. Postextrasystolic sinoatrial exit block in human sick sinus syndrome: demonstration by direct recording of sinus node electrograms. Am. Heart J. 122:1633-1643.

45. Maier, S.K., et al. 2002. An unexpected role for brain-type sodium channels in coupling of cell surface depolarization to contraction in the heart. Proc. Natl. Acad. Sci. U. S. A. 99:4073-4078.

46. Maier, S.K., et al. 2003. An unexpected requirement for brain-type sodium channels for control of heart rate in the mouse sinoatrial node. Proc. Natl. Acad. Sci. U. S. A. 100:3507-3512. 